\title{
A Biomechanical Model of Soft Tissue Deformation, with Applications to Non-rigid Registration of Brain Images with Tumor Pathology
}

\author{
Stelios K. Kyriacou and Christos Davatzikos \\ Neuroimaging Laboratory \\ Department of Radiology \\ The Johns Hopkins University School of Medicine \\ Baltimore, MD 21287
}

\begin{abstract}
The finite element method is applied to the biomechanics of brain tissue deformation. Emphasis is given to the deformations induced by the growth of tumors, and to the deformable registration of anatomical atlases with patient images. A uniform contraction of the tumor is first used to obtain an estimate of the shape of the brain prior to the growth of the tumor. A subsequent nonlinear regression method is used to improve on the above estimate. The resulting deformation mapping is finally applied to an atlas, yielding the registration of the atlas with the tumor-deformed anatomy. A preliminary $2 \mathrm{D}$ implementation that includes inhomogeneity and a nonlinear elastic material model is tested on simulated data as well as a patient image. The long-term scope of this work is its application to surgical planning systems.
\end{abstract}

Keywords: brain atlas, registration, biomechanics, inverse methods

\section{Introduction}

Much attention has been given by the medical imaging community to the modeling of normal brain anatomy. Among others, applications of anatomical modeling include computational neuroanatomy $[1,2]$, surgical path planning $[3]$, and virtual medical environments [4]. However, little attention has been given to modeling anatomical abnormalities. In this work we describe steps toward the development of a system which simulates soft tissue deformation in the brain caused by the growth of tumors. The main application of our work is currently in the non-rigid matching of brain atlases to brains with pathologies for the purposes of pre-operative planning. In particular, brain atlases can provide a wealth of information on the structural and functional organization of the brain, and ultimately on the response of different brain regions to therapeutic procedures such as radiotherapy. Since they are derived from normal brains, however, brain 
atlases must be adapted to the pathology of each individual brain. This necessitates the development of a realistic model for the mechanics of tissue deformation due to the growth of a tumor.

Recently, investigators have presented, among other topics, 2D and 3D finite element models of brain dynamics (see for example, Bandak et al. [5] and other references in the same journal issue). In the area of quasistatic brain mechanics, Nagashima and coworkers (see for example [6]), as well as Neff and coworkers [7], used 2D finite element analysis combined with poroelastic theory to allow for the movement of fluids through the brain, and a linear material and linear strains for the elastic deformations, to model edema, and hydrocephalus.

Here, we develop a method for simulating quasi-static brain mechanics, specifically investigating the growth of a brain tumor and the resulting brain deformations. Our goal is to manipulate brain atlases, which are based on normal subjects, by accounting for structural changes occurring with tumor growth and thus facilitate neurosurgical simulations for pre-operative planning and training.

\section{Methods}

We are using a plain stress finite element method i.e. we assume that there is zero stress in the direction normal to our section. Our model incorporates the parenchyma, the dura and falx membranes, and the ventricles.

\subsection{Constitutive models and parameters}

In this preliminary work, we assume that both white and gray matter and the tumor tissue are nonlinear elastic solids. The elastic properties are based on the incompressible nonlinearly-elastic neo-Hookean model with $w=\mu\left(I_{1}-3\right)$, where $w$ is the strain energy function, $\mu$ is the material constant. $I_{1}=\operatorname{tr} \mathbf{C}=$ $\lambda_{1}{ }^{2}+{\lambda_{2}}^{2}+\lambda_{3}{ }^{2}$, since $\mathbf{C}$, the Right Cauchy Green strain tensor, under a coordinate system that is based on the principal directions, may be written as: $\mathbf{C}=\operatorname{diag}\left(\lambda_{\mathbf{1}}{ }^{\mathbf{2}}, \lambda_{\mathbf{2}}{ }^{\mathbf{2}}, \lambda_{\mathbf{3}}{ }^{\mathbf{2}}\right)$, where $\lambda_{i}, i=1,2,3$ are the three principle stretches. A stretch is defined as deformed length over original length.

We use a value of $3 \mathrm{kPa}$ for the white matter $\mu$, which, for rather small values of strain [8], corresponds to a Young's modulus of $18 \mathrm{kPa}$ (a value that lies in the range of moduli given by Metz et al. [9]). In addition, we use a value of 30 $\mathrm{KPa}$ for the gray matter and tumor tissue $\mu, 10$ times higher than the white matter one.

\subsection{Normalization of the image}

Given a brain image that contains a tumor we would like to explore methods capable of calculating the origin of the tumor (the position where it started growing). We use a two-part method to perform this normalization of the image. The first part is based on simple contraction of the tumor shape, which effectively reverses the process of the tumor growth, while the second part uses the results 
of the first part and makes refinements based on nonlinear regression. The two parts of our method are explained in the following sections.

The application of this method shrinks the tumor to a small mass (ideally infinitesimal), resulting in an estimate of the normal state of the brain before the tumor growth. A normal-to-normal atlas matching procedure [10] could then be applied at this point, since two "normal" brain images are involved. Finally, the reverse procedure can grow the tumor back to its current configuration, deforming the surrounding structures accordingly. So, the final image will be a brain map that has been deformed so as to fit the patient data.

Simulation of tumor contraction In the first part, reduction of the tumor size is simulated by uniform contraction. We apply the contraction through a uniform negative strain (usually around -0.6 to -0.9 ) inside the tumor, which reduces its average diameter to approximately four tenths to one tenth of the original size respectively.

Simulation of tumor enlargement Tumor enlargement simulation is useful for two cases: 1) to create simulated tumor data for the validation of our contraction and 2) to function as the "forward" model in the nonlinear regression/optimization (see next paragraph). In the first case, the tumor seed, a circular mass of diameter about $1 \mathrm{~cm}$, is initially placed somewhere in the brain image. In the second case, the tumor seed created by the contraction run is used instead. The subsequent tumor growth is achieved by uniform expansion, similar to the contraction case above.

Nonlinear regression The second part for the tumor-bearing image normalization is based on an inverse finite element technique (in effect a nonlinear regression) required for the estimation of the original position of the brain tumor. ABAQUS [11] is used for the FEM and the Marquardt algorithm [12] is used for the nonlinear regression. We should note that the first part, the contraction, provides a mapping that may be used in itself as a rather simple image normalization map.

Since many parameters are unknown in the initial configuration, and solving for all of them might be too expensive, we have decided to optimize only the most important ones and just use a reasonable estimate for the rest of the initial parameters. For example, the undeformed shapes of the ventricles are assumed to be known from the contraction part. The shape of the tumor seed is also assumed to be the same as the one resulting from the contraction step. Three scalar parameters are considered unknown and are optimized through this inverse approach: the $\mathrm{x}$ and $\mathrm{y}$ coordinates of the centroid of the tumor seed shape and the expansion factor required for the seed to reach the tumor size observed in the final images. The errors that the regression routine minimizes against are the distances $d_{i}$ (an $N$-sized vector) of the calculated tumor nodes from the experimental (observed) tumor boundary, as well as the distances of the nodes 
on the calculated ventricles from the experimental ventricular shapes, with $N$ being the number of nodes on both tumor and ventricles. So our cost function $f$ is: $f=\sum_{i=1}^{N} d_{i}{ }^{2}$.

\section{Results}

Figure 1 depicts a simulated tumor case so that the performance of the normalization could be evaluated. The small circular black outline in panel A shows the original tumor seed before the simulated tumor expansion takes place. After the data extraction and mesh creation phase, a mesh is obtained which is overlayed in panel A. By running ABAQUS with the loads, material properties, and boundary conditions, we obtained the deformed mesh/image in panel $\mathrm{B}$. The simulated tumor of panel B was then treated as the starting point for applying the contraction part described in Section 2.2. Panel C was produced after we applied the contraction method with a suitable contraction strain found by trial and error to give an approximately same size tumor seed as the one we started with (panel A). Both the finite element (deformed) mesh and the resulting image are shown. Ideally, we would like this image to be similar to the original image of Panel A since we are using some features of it, in particular the ventricle shapes, in our regression part. Finally, panel $\mathrm{D}$ is the combination of the image in panel $\mathrm{B}$ and the ventricle outlines that correspond to the results from the nonlinear regression. Good agreement is observed.

Figures 2, and 3 represent results of applying the contraction and subsequent regression to an actual patient tumor-bearing $\mathrm{CT}$ image and to the related atlas images. Figure 2 gives the regression results. Panel A shows the patient image with a white outline denoting the tumor. Panel $\mathrm{B}$ is the superposition of the deformation mapping given by the contraction strain of -0.60 , and the subsequent regression, on the original image.

Figure 3 displays the atlas manipulations for the regression results. Panel A is the original atlas [13]. Panel B presents the warped atlas using the method described in [10] by using the overall size of the brain and ventricles. Panel $\mathrm{C}$ presents the image of panel $\mathrm{B}$ deformed with the finite element deformation mapping to obtain an atlas that has the characteristics of our patient tumor image. The vertical white lines in both panels $\mathrm{B}$ and $\mathrm{C}$ have been added to illustrate the mid-line shift due to the tumor. Finally, panel D is the patient image with a few of the structures of the atlas in panel $\mathrm{C}$ superimposed. In particular, most of the thalamic structures, the putamen, the claustrum, the cortex, and the ventricles have been included.

\section{Discussion}

The tumor contraction is easy to apply but it assumes among other things that at the post-tumor configuration, the residual stresses in the tissue are zero, something obviously not true since the tissue has already been deformed by the tumor. The regression part does not have this drawback but at the same time it does 


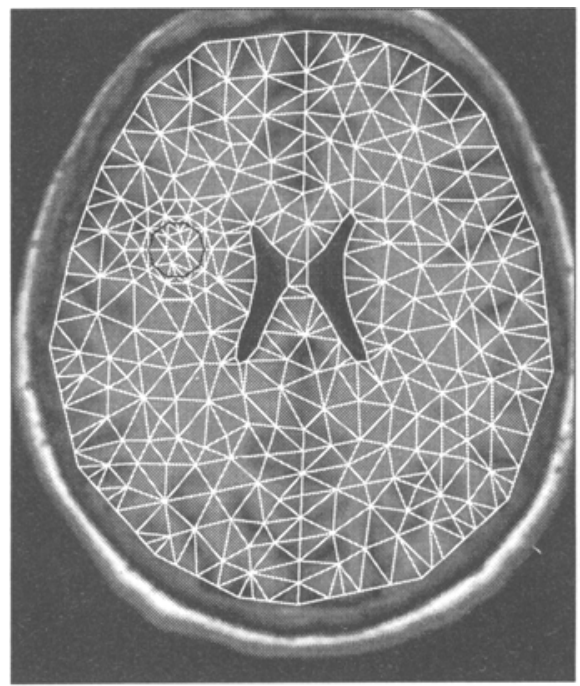

(A)

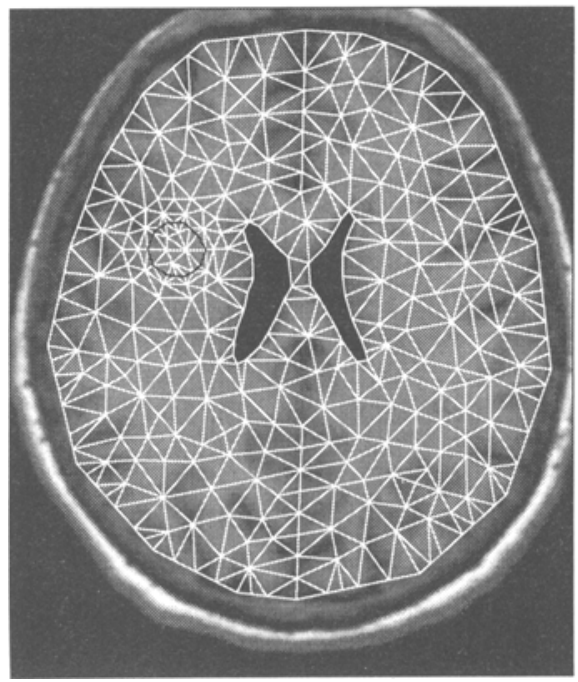

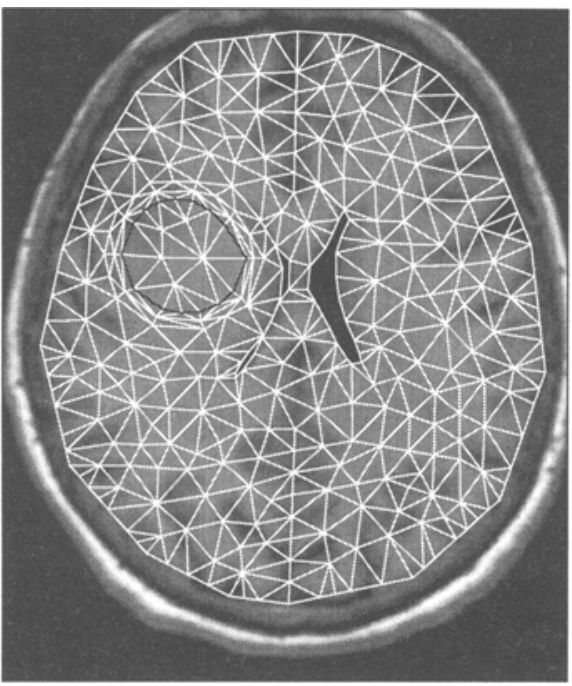

(B)

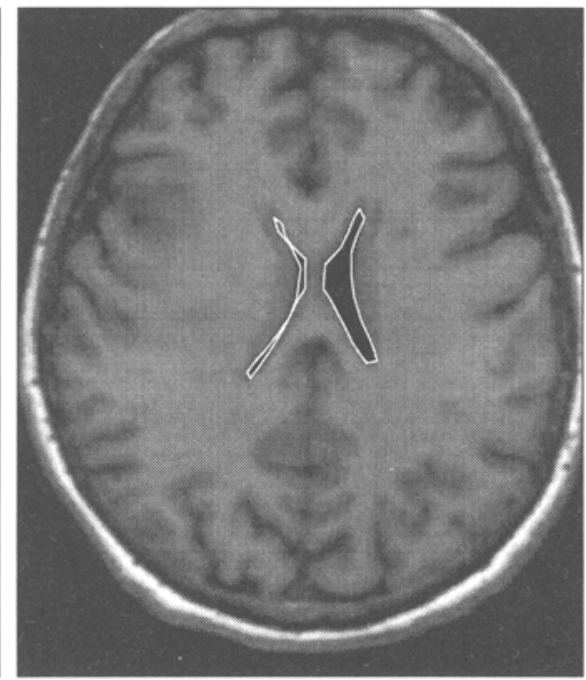

Fig. 1. Normalization of a simulated tumor: (A) the original image and mesh with the simulated tumor seed. (B) the deformed image and mesh due to a "uniform" expansion of the tumor seed. (C) The contracted image and mesh. This image corresponds to panel A. Note that the right ventricle (left side of image) is slightly larger than the corresponding one in A due to our neglect of residual stresses. (D) The ventricle outlines given by the regression overlayed on the deformed image of panel $\mathrm{B}$ to point out the correspondence. 


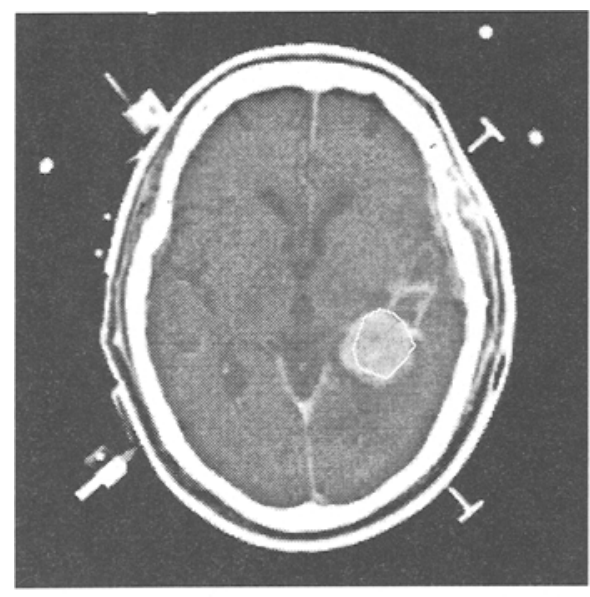

(A)

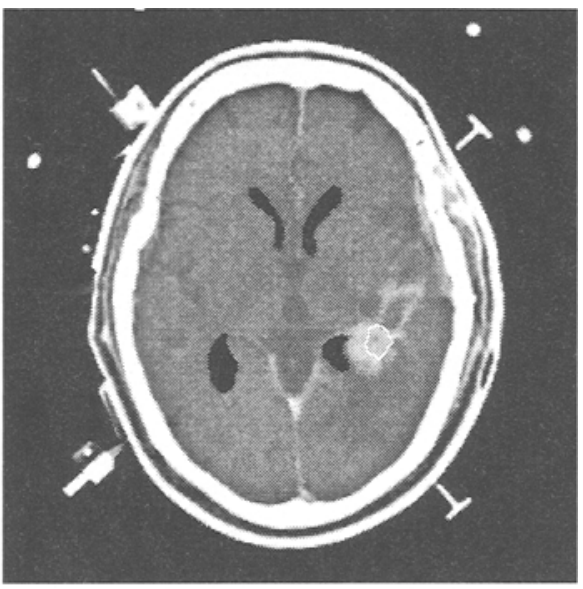

(B)

Fig. 2. Regression results for an actual tumor: CT image from a patient (panel A) with the tumor highlighted. Panel $B$ is the image created by deforming the image in panel $A$ based on the regression finite element mapping. Note that the slight mismatch between the tumor and its outline in panel $\mathrm{A}$ was needed to combat imperfections in our automatic mesh generation scheme due to the ventricle being very near the tumor.

need the undeformed configuration of the ventricles before the tumor expansion; currently this is provided by the contraction phase. We plan to overcome this limitation by using statistical information on ventricular shapes and sizes for the age and sex of the particular patient. Also we may be able to utilize the inherent symmetry of the brain for structures relatively distant to the tumor.

Our work is currently being extended from $2 \mathrm{D}$ to $3 \mathrm{D}$. The $3 \mathrm{D}$ brain volume is first segmented and the finite element mesh is then created with an automatic mesh generator, Vgrid, provided by F. Kruggel at the Max-Planck-Institute of Cognitive Neuroscience, Leipzig, Germany. Results will soon be published in a separate article.

In summary, we have shown the utility of the contraction as well as the regression techniques as a means to perform image to atlas registration for patients with localized brain deformations due to mainly tumor enlargement. The technique has potential applications in radiotherapy pre-surgical planning.

\section{Acknowledgments}

We would like to thank Dr. Don Long (Department of Neurosurgery, The Johns Hopkins Hospital), ISG Technologies (Toronto, Canada), and a grant from the American Cancer Society for partial financial support as well as Drs. R. Nick Bryan (JHU, now at NIH), and James Zinreich (JHU) for many helpful discussions, and Dr. Jeffery Williams (Department of Neurosurgery, The Johns Hopkins Hospital) for providing the CT images. 


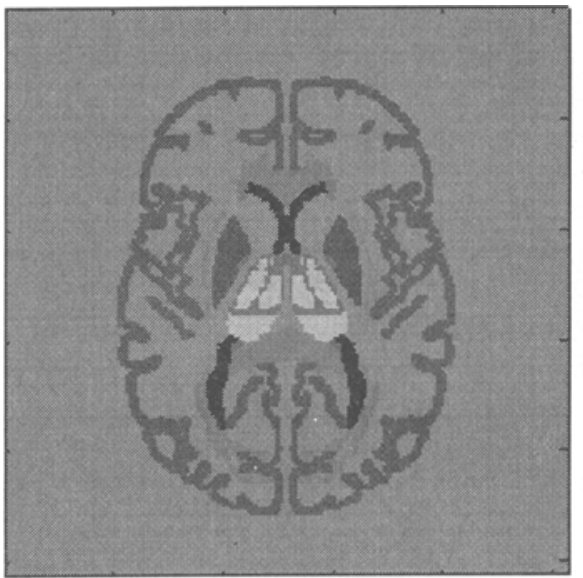

(A)

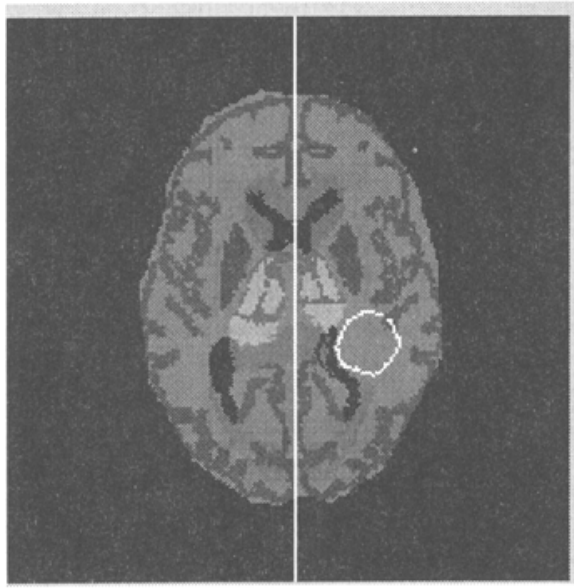

(C)

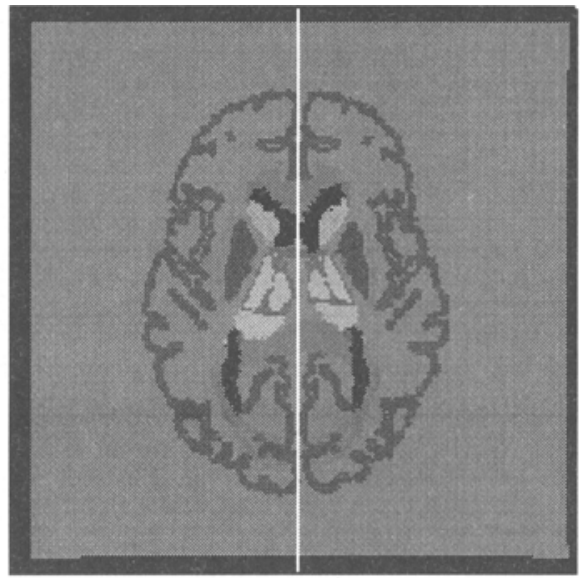

(B)

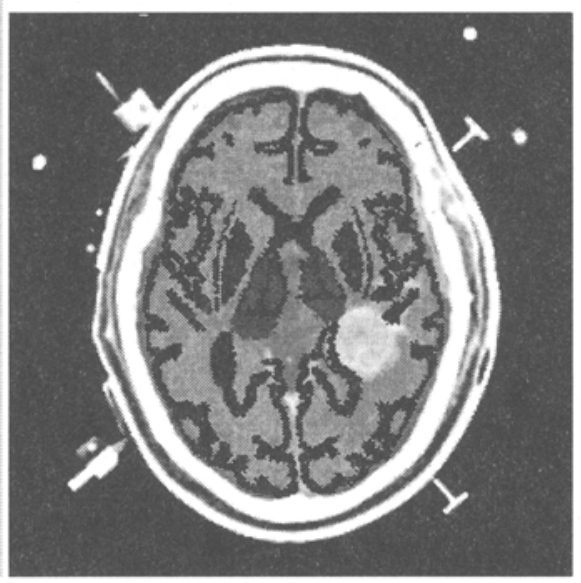

(D)

Fig. 3. Regression results for an actual tumor - atlas manipulations: (A): The original atlas slice. (B): The atlas slice warped in overall registration with the patient's image. (C): The deformation of the atlas in panel B based on the inverse of the finite element mapping of figure 2. The white outline represents the position of the tumor in the patient's image. (D): Here we have superimposed some of the atlas structures from the deformed atlas of panel $(\mathrm{C})$ on the image of figure 2, panel A. Panels B and C have been enhanced with the addition of a vertical white line to better visualize the midline shift changes. 


\section{References}

1. M.I. Miller, G.E. Christensen, Y. Amit, and U. Grenander. Mathematical textbook of deformable neuroanatomies. Proc. of the National Academy of Sciences, 90:11944-11948, 1993.

2. C. Davatzikos, M. Vaillant, S. Resnick, J.L. Prince, S. Letovsky, and R.N. Bryan. A computerized approach for morphological analysis of the corpus callosum. J. of Comp. Assisted Tomography, 20:88-97, Jan./Feb. 1996.

3. M. Vaillant, C. Davatzikos, R.H. Taylor, and R.N. Bryan. A path-planning algorithm for image guided neurosurgery. Proc. of CVRMed II - MRCAS III, pages 467-476, March 1997.

4. J. Kaye, D.N. Metaxas, and F.P. Primiano, Jr. A 3d virtual environment for modeling mechanical cardiopulmonary interactions. CVRMed-MRCAS'g7 Lecture Notes in Computer Science, Grenoble, France, 1997.

5. F.A. Bandak, M.J. Vander Vorst, L.M. Stuhmiller, P.F. Mlakar, W.E. Chilton, and J.H. Stuhmiller. An imaging-based computational and experimental study of skull fracture: finite element model development. [Review] [31 refs]. Journal of Neurotrauma, 12(4):679 88, 1995.

6. T. Nagashima, T. Shirakuni, and S.I. Rapoport. A two-dimensional, finite element analysis of vasogenic brain edema. Neurol Med Chir (Tokyo), 30(1):1-9, 1990.

7. R.P. Subramaniam, S.R. Neff, and P. Rahulkumar. A numerical study of the biomechanics of structural neurologic diseases. High Performance ComputingGrand Challenges in Computer Simulation Society for Computer Simulations, San Diego, pages 552-60, 1995.

8. K.K. Mendis, R.L. Stalnaker, and S.H. Advani. A constitutive relationship for large deformation finite element modeling of brain tissue. Journal of Biomechanical Engineering, 117(3):279-85, 1995.

9. H. Metz, J. McElhaney, and A.K. Ommaya. A comparison of the elasticity of live, dead, and fixed brain tissue. $J$ Biomech, 3(4):453-8, 1970.

10. C. Davatzikos. Spatial transformation and registration of brain images using elastically deformable models. Comp. Vision and Image Understanding, 66(2):207-222, May 1997.

11. Abaqus version 5.5. Hibbit, Karlsson, and Sorensen, Inc., USA, 1995.

12. S.K. Kyriacou, A.D. Shah, and J.D. Humphrey. Inverse finite element characterization of the behavior of nonlinear hyperelastic membranes. ASME Journal of Applied Mechanics, 64:257-62, 1997.

13. WL Nowinski, RN Bryan, and R. Raghavan (Eds.). The Electronic Clinical Brain Atlas on CD-ROM. Thieme, 1997. 